\title{
Space-Time Curvature Mode Quanta
}

\author{
Philipp Kornreich ${ }^{1,2}$ \\ ${ }^{1} 1090$ Wien, Austria \\ ${ }^{2}$ King of Prussia, PA, USA \\ Email: pkornrei@syr.edu
}

How to cite this paper: Kornreich, P. (2020) Space-Time Curvature Mode Quanta. Journal of Modern Physics, 11, 1977-1992. https://doi.org/10.4236/jmp.2020.1112125

Received: October 26, 2020

Accepted: December 15, 2020

Published: December 18, 2020

Copyright $\odot 2020$ by author(s) and Scientific Research Publishing Inc. This work is licensed under the Creative Commons Attribution International License (CC BY 4.0).

http://creativecommons.org/licenses/by/4.0/

\begin{abstract}
Einstein theorized that Gravity is not a force derived from a potential that acts across a distance. It is a distortion of space and time in which we live by masses and energy. Consistent with Einstein's theory, a model of space-time curvature modes and associated curvature quanta in slightly warped space-time generated by a light Photon is derived. Both a Schrödinger and a Second Quantized representation of the space-time curvature mode quanta are calculated and are fourth rank tensors. The eigenvalues of these equations are radii of curvature, not energy. The Eigenfunctions are linear functions of the components of the tensor that describes the curvature of space-time.
\end{abstract}

\section{Keywords}

Photons, Phonons, Gravity, General Relativity, Space-Time, Radius of Curvature, Tensor

\section{Introduction and Summary}

Consistent with Einstein's [1] description of the effect of gravity being a distortion of space and time, a model of space-time curvature modes generated by a light Photon is derived. The space-time curvature modes are derived from Einstein's Field Equation in the limit of slightly warped space-time. A Quantum Mechanical model of the space-time modes is obtained. Both a Schrödinger and a Second Quantized representation of the space-time mode quanta are derived. The eigenvalues of these equations are radii of curvature, not energy. The Eigenfunctions are linear functions of the components of the tensor that describes the curvature of space-time. Since light does not interact to first order with isotropic Minkowski space, an Earth-like mass is used to warp space-time and facilitate the interaction of the light wave and the space-time curvature modes. Flat Minkowski space-time does not have modes or quanta. Only curved space-time 
has modes and mode quanta that can interact.

The space-time curvature modes called gravity Phonons [2] are different than the Electromagnetic Field modes which also have quanta called Photons. The Electromagnetic Field modes exist in space-time. The space-time curvature modes are modes of the curvature of space-time itself. The space-time curvature modes are modes of a description of the vacuum. This derivation is only valid for slightly warped space-time. It is not valid in the vicinity of Black Holes, Neutron stars, or similar objects. Space-time in most of the Universe is only slightly curved.

The reason we don't feel or hear the space-time curvature modes is that the curvature is very slight. Even the Sun with its huge mass produces only a slight curvature in space-time. If a sphere is fitted to the space-time curvature produced by the Sun, it would need a radius equal to the distance light travels in 5 days and 3.5 hours. The radius of this sphere is the radius of curvature that characterizes the modes.

Einstein [1] theorized in 1915 that Gravity is not a force derived from a potential that acts across a distance. The effect of gravity is a distortion of space and time by masses and energy. For example, one mass moves towards another mass because it travels through space and time that is warped to make the masses travel towards each other. This has been verified by astronomical observations and experiments. Einstein also postulated the existence of wave-like space-time modes. This too has been verified by the Laser Interferometer Gravity-Wave Observatory-LIGO [3] experiment.

In this paper, equations derived from the General Relativity Theory (GRT) and verified by astronomical observations or experiments are employed at every step of the calculations.

Because this space-time curvature mode model is only valid for slightly warped space-time, it is only scale-invariant within a limited range of space-time curvature amplitudes.

The space-time curvature mode quanta are fourth rank tensors, and massless Bosons, that propagate with the speed of light. Light Photons are vector components derived from the Maxwell Electromagnetic Theory and have a spin angular momentum of 1 . The space-time curvature modes are fourth rank tensors derived from the GRT and have spin angular wave vector of 4 . The example of gravity Phonons calculated here has a very large radius of curvature of $\mathrm{R}_{\mathrm{C}}=5769.812126$ Light Years. Gravity is a very weak force. Space-time curvature modes affect the momentum and energy of objects propagating through it, and the momentum and energy of the objects affect the curvature of space-time.

Note, that this is only a mathematical model that is valid for a limited range of Nature, and in this region of Nature it is only an approximation.

The gravity Phonons, like other wave quanta, can form entangled states [4] [5]. Since gravity Phonons have radii of curvature with Astronomical dimensions and few losses, entangled gravity Phonon states can link structures over in- 


\section{terstellar distances.}

The eccentric motion of moons and planets also produces space-time wave modes. For example, the space-time wave mode generated by the Moon's eccentric orbit has a period of 27 days 7 hours, 12 minutes, and a wavelength of $7.071264665 \times 10^{14} \mathrm{~m}$ or 27.3 Light Days. Because the wavelength is much larger than the 2.667 light seconds size of the system, the motion of the Lunar system can be modeled by Newtonian Classical Mechanics.

In the late part of the 19'th century it was thought that like sound, which is a mode of the atoms in a material, electromagnetism is a mode of a substance that was named Luminiferous Aether. The Michelson-Morley experiment [6] and Einstein's calculation showed that there is no Luminiferous Aether of which electromagnetic effects are modes. Electromagnetic modes can propagate through space-time. But the electromagnetic waves are not modes of space-time.

The electromagnetic wave discussed in this paper is affected by the curvature of space-time, and the electromagnetic wave affects the curvature of space-time. If the Michelson-Morley experiment could have been performed at a higher accuracy, the interaction of the light and space-time would have been discovered. Light propagates differently in different directions due to the Earth not being an ideal sphere, and also due to the gravity effects of the Moon, the Sun, Jupiter, and the effect of other solar system objects. These effects are described by the GRT.

Sakharov [7] and Puthoff [8] derive the effect of gravity from quantum fluctuations of the vacuum. Boccaletti [9] calculated the effect of a light wave on the curvature of space-time subject to a steady electromagnetic field.

Bryce C. DeWitt [10] [11] [12] attempts to derive a Quantum theory of gravity from the GRT starting with a General Relativistic Lagrangian. His derivation is intended to be applicable to all gravity values. He wrote 3 papers but he was unable to produce a Quantum Mechanical model of gravity in a form that has useful applications.

There are a large number of publications describing unsuccessful attempts to develop models of Quantized Gravity called Gravitons [6] [7] [8] [10] [11] [12]. A number of these models use Maxwell's Equations modified by gravitational Potentials [10]. Others use Lagrangeans of Metric tensor components. This is similar to Einstein's equation of motion derived by a variational method. These run into difficulty with Gauge Normalization resulting in terms that go to infinity.

Professor Wytler C. dos Santos [13] derives the effect of gravity on electromagnetic fields expressed by the Einstein-Maxwell equation and the Rainich condition. Using the Electromagnetic Stress tensor he derives the Ricci tensor of Einstein's Field Equation. He calculates the effect of Maxwell's equation on the Riemann Curvature tensor. This derivation is in flat Minkowski space. Therefore, Professor dos Santos result is to second order in the Electromagnetic Field tensor components. The derivation in this paper uses a space-time that has a cur- 
vature due to a mass, and thus the effects calculated are to first order in the Electromagnetic Field tensor components.

Professor Rovelli [14] in his "Zakopane Lectures on Loop Gravity" describes Loop-Quantum-Gravity. He postulates Curvature Operators. These operators are similar to the operators used in this paper. The Curvature operators in this paper are derived from the Riemann Christoffel tensor and show that such operators are only valid for slight space-time curvatures. The Loop-Quantum-Gravity models space as a lattice of triangles with Plank length dimensions. Half integer spins are associated with the triangle edges. "Loop Quantum Gravity" [15] is also based on this discrete space-time model. It results in Gravity Quanta that can couple to elementary particles. This implies a very large amplitude of Gravity Quanta which is inconsistent with a weak gravity force.

The Quantum Mechanical model of Gravity derived in this paper is based on the GRT which has been verified by observations and experiments rather than use postulated quantities.

\section{Einstein's Field Equation for Slightly Curved Space-Time}

Note: Numbers such as $\{8\}$ in curly brackets denote equation numbers in the literature.

To describe the effect of the energy of the light wave on the curvature of space-time, Einstein's Field Equation is used. In Einstein's Field Equation, the Einstein tensor describes the curvature of space-time, and the Stress tensor describes the effect of the light wave energy density. The derivation is for slightly warped space-time only. The lightwave does not interact with flat isotropic Minkowski space to first order in the coordinate components.

The Metric tensor in curved space-time describes the transformation from one coordinate system to another coordinate system that is located at an infinitesimal distance away. The two coordinate systems are tilted with respect to each other. Thus, the Metric tensor describes the local tilt of the two coordinate systems. The components of the Metric tensor describe the effect of mass and energy. This is the curvature of space-time. Einstein called the Metric Tensor the Fundamental Tensor.

The components of the Metric tensor for isotropic Minkowski space are only constant for rectangular coordinates. For curvilinear coordinates, for the same isotropic space-time, the Metric tensor has components that are functions of the coordinates. Therefore, this derivation is restricted to rectangular coordinates. After the calculation, the result can be transformed into any arbitrary coordinates. The metric tensor has the following properties:
a) $\mathrm{g}^{\alpha \tau} \mathrm{g}_{\tau \beta}=\delta_{\beta}^{\alpha}$
b) $\mathrm{g}^{\alpha \tau} \mathrm{g}_{\tau \alpha}=\delta_{\alpha}^{\alpha} \quad$ therefore
c) $\mathrm{g}^{\alpha \tau} \mathrm{g}_{\tau \alpha}=4$

The Metric tensor components $g_{\mu v}$ for the case of slightly warped space-time can be approximated as follows:
a) $\mathrm{g}_{\mu v} \approx \eta_{\mu v}+\mathrm{h}_{\mu v}$ where
b) $\partial_{\alpha} \eta_{\mu v}=0$
c) $\left(\partial_{\alpha} h_{\mu v}\right)\left(\partial_{\beta} h_{\mu v}\right) \ll \partial_{\alpha} \partial_{\beta} h_{\mu v}$ 
The tensor components $h_{\mu \nu}$ are small parameters. An Earth-like mass is used to warp space-time slightly to facilitate the Photon space-time curvature mode interaction. The Metric tensor components depend on both the Earth-like mass and the energy of the light beam.

A four-dimensional Riemann space with a signature -+++ is used. It consists of the three spatial dimensions and time. The time coordinate $t$ is multiplied by the velocity of light $c$ in free space so that this coordinate, too, has dimensions of space $\mathrm{x}_{0}=\mathrm{jct}, \mathrm{x}_{1}, \mathrm{x}_{2}, \mathrm{x}_{3}$. Greek subscripts $\mu=0,1,2$, and 3 denote four-dimensional coordinates and Latin subscripts $a=1,2$, and 3 denote three-dimensional coordinates. The volume $\mathrm{V}_{4}$ and area $\mathrm{S}_{3}$ of a four-dimensional sphere is

$$
\begin{array}{ll}
\text { n Dimensions a) } V_{n}\left(R_{n}\right)=\frac{\pi^{\frac{n}{2}}}{\Gamma\left(\frac{n}{2}+1\right)} R_{n}^{n} & \text { b) } S_{n-1}\left(R_{n}\right)=\frac{d V_{n}\left(R_{n}\right)}{d R_{n}} \\
4 \text { Dimensions c) } V_{4}\left(R_{4}\right)=\frac{\pi^{2}}{2} R_{4}^{4} & \text { d) } S_{3}\left(R_{4}\right)=2 \pi^{2} R_{4}^{3}
\end{array}
$$

where $\Gamma\left(\frac{\mathrm{n}}{2}+1\right)$ is the Gamma function of $\frac{\mathrm{n}}{2}+1$. The "Surface" $S_{3}$ in fourdimensions has dimensions of volume in three-dimensional space. Here $\mathrm{R}_{4}$ is the radius of the four-dimensional sphere which is not the same as the radius $\mathrm{R}$ of the three-dimensional sphere. The Schwarzschild ratio $\frac{\mathrm{r}_{\mathrm{ss}}}{\mathrm{R}_{\oplus}}$ of the Earth-like mass is $1.390705726 \times 10^{-9}$. The ratio of the radius $\mathrm{R}_{4}$ of a four-dimensional sphere to the radius $\mathrm{R}$ of the corresponding three-dimensional sphere is constant for all sizes of the sphere. Therefore, the Schwarzschild ratio is the same for the three or four-dimensional sphere.

For a mass $\mathrm{M}$, modeled as a point mass, the radius $\mathrm{r}_{\mathrm{ss}}$ is the distance from the center of the mass $M$ to the point at which a test mass $m$ has to have an escape velocity equal to the speed of light $\mathrm{c}$. This distance is the Schwarzschild radius $\mathrm{r}_{\mathrm{ss}}$.

$$
\frac{\mathrm{r}_{\mathrm{ss}}}{\mathrm{R}_{\oplus}}=\frac{2 \mathrm{M}_{\oplus} \mathrm{G}}{\mathrm{c}^{2} \mathrm{R}_{\oplus}}
$$

where $\mathrm{M}_{\oplus}=5.9724 \times 10^{24} \mathrm{~kg}$ is the Earth-like mass. $\mathrm{r}_{\mathrm{ss}}=8.87071165 \mathrm{~mm}$ is the Schwarzschild radius [16] and $\mathrm{R}_{\oplus}=6378137 \mathrm{~m}$ is the radius of the Earth-like mass.

The $g_{00}$ component of the Metric tensor can be approximated by the effect of the time dilation calculated by Einstein [1] in the chapter "The Foundation of the GRT" section 22 "Behavior of Rods and Clocks in the Static Gravitational Field. Bending of Light Rays. Motion of the Perihelion of a Planetary Orbit." on page 160 using $\mathrm{g}_{00}$ from equation $\{70\}$. This equation for $\left\{\mathrm{g}_{00}\right\}$ is also the Schwarzschild metric [17] or Schwarzschild solution.

$$
\text { a) } \mathrm{g}_{00} \approx \frac{1}{\sqrt{1+\frac{\mathrm{r}_{\mathrm{ss}}}{\mathrm{R}_{4}}}} \quad \text { b) } 1+\mathrm{h}_{00} \approx 1-\frac{\mathrm{r}_{\mathrm{ss}}}{2 \mathrm{R}_{4}}
$$


where $\frac{\mathrm{r}_{\mathrm{ss}}}{\mathrm{R}_{4}}=1.390705726 \times 10^{-9}$ is the Schwarzschild ratio of the Earth-like mass. The distance vector with components $x_{\mu}^{\prime}$ in the vicinity of the mass $M_{\oplus}$ is approximately equal to the product of the metric tensor with components $g_{\mu v}$ times the distance vector far from the mass with components $\mathrm{x}_{v}$.

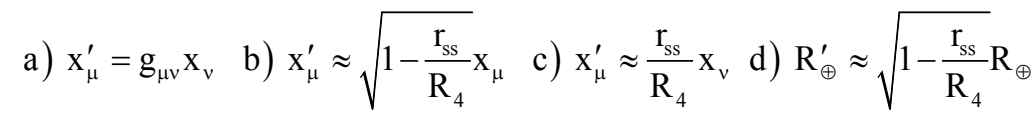

Einstein's Field equation [1] has the following form:

$$
\frac{\mathrm{c}^{4}}{8 \pi \mathrm{G}} \mathrm{G}_{\mu \nu}+\mathrm{g}_{\mu \nu} \Lambda=\mathrm{T}_{\mu \nu}
$$

Curvature of space-time $=$ Energy density

where $G_{\mu \nu}$ is a component of the Einstein tensor, $T_{\mu \nu}$ is a component of the Stress tensor and $\Lambda$ is Einstein's Cosmological Constant. The cosmological constant $\Lambda$ is neglected here. The components of the Einstein tensor $G_{\mu \nu}$ have dimensions of curvature $\frac{1}{\text { distance }^{2}}$.

The GRT has always given results in agreement with observations and experiments. Therefore the effect of the energy of the light wave on the curvature of space-time, predicted by the GRT must also be correct.

The Stress tensor has two parts. A part due to the energy density $\mathrm{u}_{\mathrm{E}}$ of the light beam and a part due to the energy density $\mathrm{u}_{\mathrm{EM}}$ of the interaction of the light beam and the mass.

The part of the Stress tensor that depends only on the energy density of the light beam is the Electromagnetic Stress tensor calculated in Appendix A. Without losing generality one can assume that the light electric field is aligned along the $\mathrm{x}_{1}$ axis and it can be assumed to be a plane wave. As is shown in Appendix A, Equation (A6), the Electromagnetic Stress Tensor [18] in this case has only 4 non zero components $\mathrm{T}_{00}$ (Light), $\mathrm{T}_{30}$ (Light), $\mathrm{T}_{03}$ (Light), and $\mathrm{T}_{33}$ (Light) all equal, to within a plus or minus sign, to the Electromagnetic Energy density $\varepsilon_{0} E_{1}^{2}$. The energy density $\mathrm{u}_{\mathrm{E}}$ of the light beam is:
a) $\mathrm{u}_{\mathrm{E}}=\mathrm{E}_{1}^{2} \varepsilon_{\mathrm{o}}$
b) $\mathrm{u}_{\mathrm{E}}=\mathrm{n} \hbar \omega$
c) $T_{\mu v}($ Light $)=e_{\mu v} n \hbar \omega$

$\mathrm{n}=1.14527 \times 10^{13} \frac{\text { Photons }}{\mathrm{m}^{3}}$ is the density of incident solar Photons at the Equator on top of the atmosphere, and $\omega=3.756087441 \times 10^{15}$ radians per second is the frequency of the light beam at the peak of the Black Body radiation curve of the Sun. The Sun's temperature is $5778^{\circ} \mathrm{K}$. The tensor components $\mathrm{e}_{\mu v}$ from Equation (A5) have the following values $\mathrm{e}_{00}=1, \mathrm{e}_{30}=-1, \mathrm{e}_{03}=-1, \mathrm{e}_{33}=1$, and all other components of this tensor are equal to zero. This term does not change with the direction of the light beam, it is symmetric, and depends on even powers of the coordinate components only. The effect of the Earth-like mass $M_{\oplus}$ on the Light electromagnetic wave with a Photon density $n$ and wave vector $\frac{\omega}{\mathrm{c}}$ is to 
deflect it. The relativistic energy density $\rho^{\prime} \mathrm{c}^{2}$ of the Earth-like mass is equal to the relativistic mass times the square of the velocity of light divided by the surface $\mathrm{S}_{3}$ of a four-dimensional sphere. Using Equation (4) for $\frac{r_{s s}}{R_{4}}$ one obtains
a) $\rho^{\prime} c^{2}=\frac{M_{\oplus} c^{2}}{S_{3} \sqrt{1-\frac{r_{s s}}{R_{4}}}}$
b) $\rho^{\prime} c^{2} \approx \frac{M_{\oplus} c^{2}}{2 \pi^{2} R_{4}^{3}}\left(1+\frac{r_{s s}}{2 R_{4}}+\cdots\right)$
c) $\rho^{\prime} c^{2} \approx \frac{M_{\oplus} c^{2}}{2 \pi^{2} R_{4}^{3}}+\frac{M_{\oplus}^{2} G}{2 \pi^{2} R_{4}^{4}}+\cdots$
where
d) $r_{s s}=\frac{2 M_{\oplus} G}{c^{2}}$

By using Equation (8b) and the second term on the right of Equation (9c) to form the interaction energy density $\mathrm{u}_{\mathrm{EM}}$ of the light beam and the Earth-like mass:
a) $u_{E M}=\sqrt{\frac{M_{\oplus}^{2} G}{2 \pi^{2} R_{4}^{4}} n \hbar \omega}$
b) $\mathrm{T}_{\mu v}=\mathrm{e}_{\mu \nu} \sqrt{\frac{\mathrm{M}_{\oplus}^{2} \mathrm{G}}{2 \pi^{2} \mathrm{R}_{4}^{4}} \mathrm{n} \hbar \omega}$

The energy density $\mathrm{u}_{\mathrm{EM}}$ of the interaction of the light beam and the space-time curvature modes are not symmetric and can be a linear function of the coordinates. The much smaller energy density $u_{E}$ of the light beam does not depend on the direction of the light beam. It is symmetric and depends to second-order on the coordinates. It has been neglected.

$$
\mathrm{u}_{\mathrm{EM}}=1616.138805 \frac{\text { Joules }}{\mathrm{m}^{3}} \quad \mathrm{u}_{\mathrm{E}}=4.536471695 \times 10^{-6} \frac{\text { Joules }}{\mathrm{m}^{3}}
$$

The radius $R_{4}$ of the four-dimensional sphere is calculated from the radius $R_{\oplus}$ of the three-dimensional sphere by setting the $S_{3}$ equal to the three-dimensional volume of the sphere. Substituting Equation (10b) into Einstein's Field Equation, Equation (7).

$$
\frac{\mathrm{c}^{4}}{8 \pi \mathrm{G}} \mathrm{G}_{\mu v}=\mathrm{e}_{\mu \nu} \sqrt{\frac{\mathrm{M}_{\oplus}^{2} \mathrm{G}}{2 \pi^{2} \mathrm{R}_{4}^{4}} \mathrm{n} \hbar \omega}
$$

The Einstein Tensor with components $G_{\mu \nu}$ is a function of the Ricci tensor with components $R_{\mu v}$ and the scalar curvature $R_{\alpha \alpha}$.

$$
\mathrm{G}_{\mu v}=\mathrm{R}_{\mu v}-\frac{1}{2} \mathrm{R}_{\alpha \alpha} \mathrm{g}_{\mu \nu}
$$

The Ricci tensor with components $R_{\mu v}$ is equal to the contraction over the indices $\rho$ and $\tau=\rho$ of the Riemann-Christoffel tensor with components $B_{\mu v \tau}^{\rho}$. The Riemann-Christoffel tensor with components $B_{\mu v \tau}^{\rho}$ describes the curvature of space-time. It describes the transport $\mathrm{B}_{\mu \nu \tau}^{\rho} \mathrm{V}_{\rho}$ of a covariant vector with components $V_{\rho}$ around an infinitesimal size volume in curved space-time. The difference of the vector $\mathrm{V}$ when transported clockwise and counter-clockwise back to the same point is a measure of the curvature of space-time. Therefore, the Riemann-Christoffel tensor is equal to the difference between two similar tensors $\mathrm{A}_{\mu \nu \tau}^{\rho}$ and $\mathrm{A}_{\mu \tau \nu}^{\rho}$ shown in reference [1] in section 12 "The Riemann-Christoffel Tensor", equation $\{42\}$ and the preceding discussion. The route of the vector can 
be modeled by a path with four turning points. The index $\mu$ is the number of each turning point. At each turning point $\mu$, a rotation matrix associated with a clockwise path has indices $\nu \tau$, and the rotation matrices associated with the counter-clockwise path have indices $\tau$.

The first Riemann-Christoffel tensor contravariant index describes the index of the components $\rho$ of the vector V. The second Riemann-Christoffel tensor covariant index describes the turning point number $\mu$. The row and column numbers $v$ and $\tau$ of the rotation matrices at the turning points provide another two covariant Riemann-Christoffel tensor indices. Thus, the Riemann-Christoffel tensor $\mathrm{B}_{\mu \nu \tau}^{\rho}$ is a fourth-rank tensor with 4 indices $\rho, \mu, \nu$ and $\tau$. In a flat Minkowski space, the vector $\mathrm{V}$ has the same form after being transported around the infinitesimal volume clockwise or counter-clockwise and thus, the Riemann-Christoffel tensor is equal to zero.

The Riemann-Christoffel tensor is also equal to the commutation relation of two covariant derivatives [1]. In flat space, the covariant derivatives reduce to ordinary derivatives that commute. Thus, in flat Minkowski space, the commutation relation of two derivatives is equal to zero, and therefore, the Riemann-Christoffel tensor is equal to zero.

This description has too much unnecessary data to just describe the curvature of the space-time. Certainly, the initial direction of the vector V with component indices $\rho$ is irrelevant. It is, also, sufficient to describe the new direction acquired by the vector $\mathrm{V}$ at each turning point by a vector with index $v$ or $\tau$, rather than describing the turning process by a matrix with indices $v$ and $\tau$. This leaves the tensor describing the curvature of space-time with just two indices, $\mu$ and $\nu$. Thus, the Riemann-Christoffel tensor describing the curvature of space-time can be reduced to a second-rank tensor, the Ricci tensor with indices $\mu$ and $\nu$. The conversion of the fourth-rank Riemann-Christoffel tensor to the second-rank Ricci tensor, with components $R_{\mu \nu}$ can be accomplished by taking the inner product of the vector $\mathrm{V}$ with components $\mathrm{V}_{\rho}$ and a vector with components that have indices equal to the row numbers $\tau$ of the matrices at the turning points. A vector with components with indices equal to the column numbers $v$ of the matrices at the turning points can also be used. Thus, the Riemann-Christoffel tensor is contracted over the two indices $\rho$ and $\tau$ to obtain the Ricci tensor $\mathrm{R}_{\mu \nu}$.

$$
\mathrm{R}_{\mu v}=\mathrm{B}_{\mu \nu \rho}^{\rho}
$$

The Riemann-Christoffel tensor was derived in Reference [1], page 141 equation $\{43\}$ :

$$
\begin{aligned}
& \text { a) } \mathrm{B}_{\mu v \tau}^{\rho}=-\partial_{\tau} \Gamma_{\mu \nu}^{\rho}+\partial_{v} \Gamma_{\mu \tau}^{\rho}-\Gamma_{\mu \nu}^{\alpha} \Gamma_{\alpha \tau}^{\rho}+\Gamma_{\mu \tau}^{\alpha} \Gamma_{\alpha \nu}^{\rho} \\
& \text { b) } \mathrm{B}_{\mu \nu \tau}^{\rho} \approx-\partial_{\tau} \Gamma_{\mu \nu}^{\rho}+\partial_{v} \Gamma_{\mu \tau}^{\rho} \quad \text { where } \partial_{\alpha} \partial_{\beta} \mathrm{h}^{\alpha \beta} \gg\left(\partial_{\mu} \mathrm{h}^{\alpha \beta}\right)\left(\partial_{\alpha} \mathrm{h}^{\mu \beta}\right) \\
& \text { c) } \mathrm{R}_{\mu v} \approx-\partial_{\rho} \Gamma_{\mu \nu}^{\rho}+\partial_{v} \Gamma_{\mu \rho}^{\rho}
\end{aligned}
$$

The Christoffel symbol $\Gamma_{\mu \nu}^{\rho}$ is a function of the derivatives of the Metric tensor components. The Metric tensor components are of the order of the Schwarzschild 
ratio $\frac{\mathrm{r}_{\mathrm{sS}}}{\mathrm{R}}=1.3907 \times 10^{-9}$. Therefore, the last two terms of Equation (14a) consisting of products of Christoffel symbols are of the order of $1.934 \times$ $10^{-18}$, and thus can be neglected which simplifies the calculation. The Christoffel symbols $\Gamma_{\mu v}^{\rho}$ are obtained from reference [1] Einstein's Equation of Motion, equation $\{20 \mathrm{~d}\}$ and equations $\{21\}$ and $\{23\}$ on page 182 , given here by:

$$
\begin{aligned}
& \text { a) } \Gamma_{\mu v}^{\rho}=-\frac{1}{2} \mathrm{~g}^{\rho \alpha}\left[\partial_{v} \mathrm{~g}_{\mu \alpha}+\partial_{\mu} \mathrm{g}_{v \alpha}-\partial_{\alpha} \mathrm{g}_{\mu v}\right] \\
& \text { b) } \Gamma_{\mu \nu}^{\rho} \approx-\frac{1}{2} \eta^{\rho \alpha}\left[\partial_{v} \mathrm{~h}_{\mu \alpha}+\partial_{\mu} \mathrm{h}_{v \alpha}-\partial_{\alpha} \mathrm{h}_{\mu \nu}\right]
\end{aligned}
$$

where $\eta^{\rho \alpha}$ is a Minkowski Metric tensor component for a Riemann space with a signature -+++ . Note from Equations (15) that the Christoffel symbol components are functions of the derivatives of the Metric tensor components. The Metric tensor components $h_{\mu \nu}$ shown in Equations (1) and (2) are very small. For slightly warped space-time the Metric tensor components $g_{\mu v}$ of Equation (2) can be approximated by the Minkowski tensor components $\eta_{\mu v}$. Substituting Equation (15b) into Equation (14c)

$$
\mathrm{R}_{\mu v} \approx \frac{1}{2} \eta^{\rho \alpha}\left(\partial_{\rho} \partial_{\mu} \mathrm{h}_{v \alpha}-\partial_{\rho} \partial_{\alpha} \mathrm{h}_{\mu \nu}-\partial_{v} \partial_{\mu} \mathrm{h}_{\rho \alpha}+\partial_{\rho} \partial_{\alpha} \mathrm{h}_{\mu \rho}\right)
$$

The trace of the Ricci tensor can be calculated from Equation (16)

$$
\frac{1}{2} \mathrm{R}_{\gamma} \approx \frac{1}{4} \eta^{\rho \alpha}\left[\partial_{\rho} \partial_{\gamma} \mathrm{h}_{\gamma \alpha}-\partial_{\rho} \partial_{\alpha} \mathrm{h}_{\gamma \gamma}-\partial_{\gamma} \partial_{\gamma} \mathrm{h}_{\rho \alpha}+\partial_{\gamma} \partial_{\alpha} \mathrm{h}_{\gamma \rho}\right]
$$

Substituting Equation (16) and Equation (17) into Equation (12).

$$
\begin{aligned}
\mathrm{G}_{\mu \nu}= & \frac{1}{2} \eta^{\rho \alpha}\left(\partial_{\rho} \partial_{\mu} \mathrm{h}_{\mu \alpha}-\partial_{\rho} \partial_{\alpha} \mathrm{h}_{\mu \nu}-\partial_{v} \partial_{\mu} \mathrm{h}_{\rho \alpha}+\partial_{v} \partial_{\alpha} \mathrm{h}_{\mu \rho}\right) \\
& -\frac{1}{4} \eta^{\mu \nu} \eta^{\rho \alpha}\left(\partial_{\rho} \partial_{\beta} \mathrm{h}_{\beta \alpha}-\partial_{\rho} \partial_{\alpha} \mathrm{h}_{\beta \beta}-\partial_{\beta} \partial_{\beta} \mathrm{h}_{\rho \alpha}+\partial_{\beta} \partial_{\alpha} \mathrm{h}_{\beta \rho}\right)
\end{aligned}
$$

Equation (18) for Einstein's tensor can be written in terms of a fourth-rank tensor with components $\mathrm{s}_{\mu \mathrm{v}}^{\alpha \beta}$.

$$
\partial_{\alpha} \partial_{\beta} s_{\mu v}^{\alpha \beta}=G_{\mu v}
$$

Since the gradient vector with components $\partial_{\alpha}$ is covariant and the inner product $\partial_{\alpha} \partial_{\beta} s_{\mu v}^{\alpha \beta}$ contracts to $G_{\mu v}$, the tensor with components $s_{\mu v}^{\alpha \beta}$ must be partially contravariant. The tensor components $s_{\mu \nu}^{\alpha \beta}=s_{\mu v}^{\alpha \beta}\left(h_{\rho \tau}\right)$ are linear functions of the Metric tensor components. For example, the $\mathrm{G}_{33}$ component of the Einstein tensor derived from Equation (18) is:

$$
\mathrm{G}_{33} \approx \frac{1}{2}\left[\begin{array}{llll}
\partial_{0} & \partial_{1} & \partial_{2} & \partial_{3}
\end{array}\right]\left[\begin{array}{cccc}
\mathrm{h}_{33} & 0 & 0 & -\mathrm{h}_{03} \\
0 & \mathrm{~h}_{22} & -\mathrm{h}_{21} & 0 \\
0 & -\mathrm{h}_{12} & \mathrm{~h}_{11} & 0 \\
-\mathrm{h}_{30} & 0 & 0 & \mathrm{~h}_{00}
\end{array}\right]\left[\begin{array}{c}
\partial_{0} \\
\partial_{1} \\
\partial_{2} \\
\partial_{3}
\end{array}\right]
$$

After multiplying out Equation (20) all derivatives have to be moved to the left like in Equation (19). The differentiation operators $\partial_{\mu}$ operate on the Metric tensor components that describe the effect of the Earth-like mass and the light 
electromagnetic field. Substituting Equation (10b) and Equation (19) into Equation (7) to obtain for Einstein's Field Equation in the limit of slightly warped space-time.

$$
\begin{aligned}
& \text { а) } \frac{c^{4}}{8 \pi G}\left(\partial_{\alpha} \partial_{\beta} s_{\mu v}^{\alpha \beta}+\partial_{\alpha}^{\text {Earth }} \partial_{\beta}^{\text {Earth }} s_{\mu v}^{\alpha \beta}\right)=e_{\mu v} \sqrt{\frac{M_{\oplus}^{2} G}{2 \pi^{2} R_{4}^{4}} n \hbar \omega} \\
& \text { в) } \frac{c^{4}}{8 \pi G}\left(\partial_{\alpha} \partial_{\beta} s_{\mu \nu}^{\alpha \beta}+K_{\alpha} K_{\beta} \beta_{\mu \nu}^{\alpha \beta}\right)=e_{\mu v} \sqrt{\frac{M_{\oplus}^{2} G}{2 \pi^{2} R_{4}^{4}} n \hbar \omega}
\end{aligned}
$$

Equation (21) has dimensions of energy density. Equation (21b) is in the form of a Hamiltonian. The canonical variables are the momentum components $-\mathrm{j} \partial_{\alpha}$ and the curvature vector components $\boldsymbol{K}_{\alpha}$. The first term $\partial_{\alpha} \partial_{\beta} \mathrm{s}_{\mu v}^{\alpha \beta}$ of Equation (21a) describes the part associated with the effect of the interaction energy density of the light beam and the Earth-like mass.

The terms $\partial_{\alpha}^{\text {Earth }} \partial_{\beta}^{\text {Earth }} \mathrm{s}_{\mu v}^{\alpha \beta}=\boldsymbol{K}_{\alpha} \boldsymbol{K}_{\beta} \mathrm{s}_{\mu \nu}^{\alpha \beta}$ appearing in Equation (21a) and Equation (21b) describe the part associated with the curvature of space-time due to the Earth-like mass. This is similar to equation $\{7\}$ in "Physical and Geometric Interpretation of the Ricci Tensor and Scalar Curvature" by L. C. Loveridge [19]. The tensor with components $s_{\mu v}^{\alpha \beta}$ is dimensionless.

\section{Schrödinger Formulation of Space-Time Curvature Modes}

Equation (21b), derived from the GRT, bears a resemblance to a tensor form of Schrödinger's equation. Here $-\mathrm{j} \partial_{\alpha}$ is a component of a momentum vector operator and $\boldsymbol{K}_{\alpha}$ is a curvature vector potential operator. $\boldsymbol{K}_{\alpha}$ stands for Krümmung, curvature in German. By multiplying Equation (21b) by $\frac{8 \pi \mathrm{G}}{\mathrm{c}^{4}}$ one obtains a tensor Schrödinger like equation for curvature instead of energy. The equation instead of having an energy Eigenvalue, as the conventional Schrödinger equation, this equation has a curvature Eigenvalue $\frac{1}{\mathrm{R}_{\mathrm{EM}}^{2}}$.

$$
\partial_{\alpha} \partial_{\beta} \psi_{\mu \nu}^{\alpha \beta}+V_{\alpha \beta} \psi_{\mu \nu}^{\alpha \beta}=\frac{\eta_{\alpha \beta}}{R_{E M}^{2}} \psi_{\mu \nu}^{\alpha \beta}
$$

$\eta_{\alpha \beta}$ are components of the Minkowski Metric tensor and $\psi_{\mu \nu}^{\alpha \beta}\left(\mathrm{h}_{\lambda \rho}\right)$ is a dimensionless tensor wave function of a space-time curvature mode. The tensor wave functions $\psi_{\mu \nu}^{\alpha \beta}\left(h_{\lambda_{\rho}}\right)$ are linear functions of the components of the Metric tensor components $h_{\lambda \rho}$ that describe the curvature of space-time due to the light beam and Earth-like mass. The eigenvalues of these equations are radii of curvature, not energy.

$$
\text { a) } \psi_{(\mu v)}^{\alpha \beta}=\mathrm{ws}_{\mu v}^{\alpha \beta} \quad \text { b) } 1=\frac{1}{\mathrm{~V}_{4 \text {-Volume }}} \int_{\text {4-Volume }} \psi_{\alpha \beta(\mu v)}^{*} \psi_{(\mu v)}^{\alpha \beta} \mathrm{d}^{4} \mathrm{x}
$$

No summation over subscripts in brackets is not implied. This derivation of a Quantum Mechanical formulation is only valid for slightly curved space-time. Since this is a straightforward derivation from Einstein's Field Equation no 
Gauge requirements are necessary. From Equation (21) the square of the reciprocal of the radius of curvature $\mathrm{R}_{\mathrm{EM}}$ is:

$$
\frac{1}{\mathrm{R}_{\mathrm{EM}}^{2}}=\mathrm{e}_{\mu v} \frac{8 \pi \mathrm{G}}{\mathrm{c}^{4}} \sqrt{\frac{\mathrm{M}_{\oplus}^{2} \mathrm{G}}{2 \pi^{2} \mathrm{R}_{4}^{4}} \mathrm{n} \hbar \omega}
$$

where $\mathrm{R}_{\mathrm{EM}}=5.45866374 \times 10^{19} \mathrm{~m}$ or $\mathrm{R}_{\mathrm{EM}}=5769.812126$ Light Years.

\section{Formulation Second Quantized Form of Space-Time Curvature Modes}

Dividing Equation (21b) by $\sqrt{\frac{M_{\oplus}^{2} G}{2 \pi^{2} R_{4}^{4}} n \hbar \omega}$, using Equation (24) in the resulting equation and expanding the ensuing equation.

$$
\mathrm{e}_{\mu v}=\frac{1}{2}\left[\left(\boldsymbol{K}_{\alpha}+\mathrm{j} \partial_{\alpha}\right)\left(\boldsymbol{K}_{\beta}-\mathrm{j} \partial_{\beta}\right) \mathrm{R}_{\mathrm{M}}^{2} \mathrm{~s}_{\mu v}^{\alpha \beta}+\left(\boldsymbol{K}_{\alpha}-\mathrm{j} \partial_{\alpha}\right)\left(\boldsymbol{K}_{\beta}+\mathrm{j} \partial_{\beta}\right) \mathrm{R}_{\mathrm{M}}^{2} \mathrm{~s}_{\mu v}^{\alpha \beta}\right]
$$

The j $\partial_{\alpha}$ and $\boldsymbol{K}_{\alpha}$ curvature operators don't commute.

$$
-j \partial_{\alpha} K_{\beta} R_{\mathrm{EM}}^{2} s_{\mu \nu}^{\alpha \beta}+j K_{\alpha} \partial_{\beta} R_{\mathrm{EM}}^{2} s_{\mu \nu}^{\alpha \beta}=e_{\mu v}
$$

Similar to the derivation of the quantization of the Electromagnetic Field by G. M. Wysin [20] in equations $\{43\}$ and $\{44\}$ the state raising and state lowering operators are defined.

$$
\begin{aligned}
& \text { a) } \boldsymbol{b}_{\alpha}^{\dagger} \exp \left(-\mathrm{jq}_{\mathrm{a}} \mathrm{x}_{\mathrm{a}}\right) \equiv \frac{\mathrm{R}_{\mathrm{ME}}}{\sqrt{2}}\left(\boldsymbol{K}_{\alpha}-\mathrm{j} \partial_{\alpha}\right) \\
& \text { b) } \boldsymbol{b}_{\alpha} \exp \left(\mathrm{jq}_{\mathrm{a}} \mathrm{x}_{\mathrm{a}}\right) \equiv \frac{\mathrm{R}_{\mathrm{ME}}}{\sqrt{2}}\left(\boldsymbol{K}_{\alpha}+\mathrm{j} \partial_{\alpha}\right)
\end{aligned}
$$

The state raising and state lowering operators are dimensionless curvature operators. Here $\exp \left(-\mathrm{jq}_{\tau} \mathrm{x}_{\tau}\right)$ represents a forward traveling space-time wave mode and $\exp \left(\mathrm{jq}_{\tau} \mathrm{x}_{\tau}\right)$ represents a reverse traveling space-time wave mode. The gravity Phonon number state wave functions $\left|\mathrm{m}_{\lambda}\right\rangle$ form a Hilbert space. The state raising vector component operators $\boldsymbol{b}_{\alpha}^{\dagger}$ and state lowering vector component operators $\boldsymbol{b}_{\alpha}$ are operators on the gravity Phonon Hilbert space.

$$
\begin{aligned}
& \text { a) } \boldsymbol{b}_{\alpha} \mathrm{s}_{\mu v}^{\alpha \beta}\left|\mathrm{m}_{\lambda}\right\rangle=\hat{\mathrm{a}}_{\alpha} \mathrm{s}_{\mu v}^{\alpha \beta} \sqrt{\mathrm{m}}\left|\left(\mathrm{m}_{\lambda}-1\right)\right\rangle \\
& \text { b) } \boldsymbol{b}_{\alpha} \mathrm{s}_{\mu \nu}^{\alpha \beta}\left|0_{\lambda}\right\rangle=0 \\
& \text { c) } \boldsymbol{b}_{\alpha}^{\dagger} \mathrm{s}_{\mu \nu}^{\alpha \beta}\left|\mathrm{m}_{\lambda}\right\rangle=\hat{\mathrm{a}}_{\alpha} \mathrm{s}_{\mu \nu}^{\alpha \beta} \sqrt{\mathrm{m}+1}\left|\left(\mathrm{~m}_{\lambda}+1\right)\right\rangle
\end{aligned}
$$

$\hat{\mathrm{a}}_{\beta}$ is a component of a unit vector in four dimensional space. The commutation relations of the $\boldsymbol{b}_{\alpha}$ and $\boldsymbol{b}_{\beta}^{\dagger}$ are:

$$
\begin{aligned}
& \boldsymbol{b}_{\beta} \boldsymbol{b}_{\alpha}^{\dagger}-\boldsymbol{b}_{\beta}^{\dagger} \boldsymbol{b}_{\alpha}=\mathrm{e}_{\mu v} \\
& \text { where } \boldsymbol{b}_{\beta} \boldsymbol{b}_{\alpha}^{\dagger}=\boldsymbol{b}_{\alpha}^{\dagger} \boldsymbol{b}_{\beta} \text { for } \alpha \neq \beta
\end{aligned}
$$

Substituting Equation (27) into Equation (25) and dividing the result equation by $\mathrm{R}_{\mathrm{EM}}^{2}$. 


$$
\boldsymbol{C}_{\mu v}=\frac{1}{2 \mathrm{R}_{\mathrm{EM}}^{2}}\left(\boldsymbol{b}_{\alpha} \boldsymbol{b}_{\beta}^{\dagger}+\boldsymbol{b}_{\alpha}^{\dagger} \boldsymbol{b}_{\beta}\right)
$$

where $\boldsymbol{C}_{\mu v}$ is a curvature Hamiltonian tensor. Since the Metric tensor is symmetric, the tensor with components $s_{\mu \nu}^{\alpha \beta}=s_{\mu \nu}^{\beta \alpha}$ and $s_{\mu v}^{\alpha \beta}=s_{v \mu}^{\alpha \beta}$ is also symmetric. The tensor with components $s_{\mu v}^{\alpha \beta}$ is a linear function the components $\mathrm{h}_{\alpha \beta}=\mathrm{h}_{\beta \alpha}$ of the Metric tensor. Substituting Equation (29) into Equation (30) to obtain a curvature Hamiltonian tensor $\boldsymbol{C}_{\mu v}$ in second quantized form for the gravity Phonons is:

$$
\boldsymbol{C}_{\mu v}=\frac{1}{\mathrm{R}_{\mathrm{EM}}^{2}}\left(\boldsymbol{b}_{\alpha}^{\dagger} \boldsymbol{b}_{\beta} \mathrm{s}_{\mu \nu}^{\alpha \beta}+\frac{1}{2} \mathrm{e}_{\mu v}\right)
$$

The gravity Phonon ground state from Equation (31) is:

$$
\begin{aligned}
& \text { a) }\left\langle 0_{\lambda}\left|\boldsymbol{C}_{\mu v}\right| 0_{\lambda}\right\rangle=\frac{1}{\mathrm{R}_{\mathrm{EM}}^{2}}\left\langle 0_{\lambda}\left|\left(\boldsymbol{b}_{\alpha}^{\dagger} \boldsymbol{b}_{\beta} \mathrm{s}_{\mu \nu}^{\alpha \beta}+\frac{1}{2} \mathrm{e}_{\mu \nu}\right)\right| 0_{\lambda}\right\rangle \\
& \text { b) }\left\langle 0_{\lambda}\left|\boldsymbol{C}_{\mu \nu}\right| 0_{\lambda}\right\rangle=\frac{1}{2 \mathrm{R}_{\mathrm{EM}}^{2}} \mathrm{e}_{\mu \nu}
\end{aligned}
$$

The tensor wave functions that describe the components of the Metric tensor can be calculated from the Curvature Hamiltonians of Equation (22) and Equation (31). The Metric tensor components describe the curvature of space-time due to the Earth-like mass and the energy of the light beam.

\section{Conclusions}

Both a Schrödinger and second quantized formulations of space-time curvature quanta are derived. The tensor wave functions that describe the components of the Metric tensor can be calculated from the Curvature Hamiltonians. The Metric tensor components describe the effect of the Earth-like mass and the energy of the light beam.

Einstein [1] theorized in 1915 that Gravity is not a force derived from a potential that acts across a distance. Gravity is a distortion of space and time by masses and energy. For example, a test mass moves towards another mass because it travels through space and time that is warped in such a way as to make the test mass travel towards the other mass. Einstein also postulated the existence of wave-like space-time modes. These too, have been verified by the LIGO experiment [3].

In this paper, equations derived from the GRT and verified by astronomical observations or experiments are employed at every step of the calculations.

A model of space-time curvature modes in slightly warped space-time generated by a light Photon is derived. Since an electromagnetic wave does not interact with isotropic space-time, to first order, an Earth-like mass is used to warp space-time to facilitate the Photon space-time curvature mode interaction. Each space-time curvature mode is a fourth-rank tensor and can have any number of curvature quanta, called gravity Phonons. Gravity Phonons have very large radii 
of curvature in agreement with gravity being a weak force. The gravity Phonons are fourth-rank tensors, massless Bosons, have a spin angular wave vector of 4 , and propagate with the speed of light. Energy and momentum are conserved because curved space-time causes a change in energy and momentum of the electromagnetic wave, and the energy of the electromagnetic wave causes a change of the curvature of space-time. Because this model is only valid for slightly warped space-time, it is only scale invariant within a limited range of space-time curvature amplitudes.

A large number of publications describing unsuccessful attempts to develop models of Quantized Gravity called Gravitons have been published. A number of these models use Post Newtonian Maxwell's Equations modified by gravitational potentials. These run into difficulty with Gauge Normalization resulting in terms that go to infinity. The other approach uses "Loop Quantum Gravity". It uses a lattice of triangles with Plank distance and Plank time sides to calculate the gravity energy quanta. This results in huge energy quanta amplitudes, not consistent with gravity being a very weak force. Therefore, in order to derive a Quantum Mechanical model of Gravity, one has to calculate space-time curvature mode quanta rather than use Gravitational potentials or Plank space and time to formulate gravity energy quanta.

Space-time is only slightly warped throughout most of the Universe except in the vicinity of Black Holes and Neutron Stars. For example, space-time at the surface of the sun has a radius of curvature of approximately 5.165 Light Days. Thus, gravity Phonons are a suitable Quantum Mechanical description of most of space-time in the Universe.

The space-time curvature quanta, like electromagnetic wave quanta, can form entangled states [4] [5]. Photons are vector quantities while gravity Phonons are fourth-rank tensors. Gravity Phonons have radii of curvature with Astronomical dimensions, therefore entangled gravity Phonon fourth-rank tensor states can link structures over interstellar distances.

\section{Acknowledgements}

I thank my wife Marlene Danzig Kornreich for her suggestions to the text, and for making the text more understandable to a reader. I also thank her for editing and working together on this manuscript.

\section{Conflicts of Interest}

The author declares no conflicts of interest regarding the publication of this paper.

\section{References}

[1] Einstein, A., Lorentz, H.A., Weyl, H. and Minkowski, H. (1952) The Principles of Relativity. Dover Publishing Co., Mineola.

[2] Kornreich, P. (2019) Journal of Modern Physics, 10, 1674-1695. 
[3] Abbott, B.P., et al. (2016) Physical Review Letters, 116, Article ID: 061102.

[4] Liao, S.-K., Young, H.-L., Liu, C., Shentu, G.-L., Li, D.-D., Lin, J., Dai, H., Zhao, S.-Q., Li, B., Guan, J.-Y., Chen, W., Lin, Y.-H., Pan, G.-S., Pelc, J.S., Fejer, M.M., Liu, W.-Y., Yin, J., Ren, J.-G., Wang, X.-B., Zhang, Q., Peng, C.-Z. and Pan, J.-W. (2017) Nature Photonics, 11, 509-513. https://doi.org/10.1038/nphoton.2017.116

[5] Krenn, M., Malik, M., Erhard, M. and Zeilinger, A. (2017) Philosophical Transactions of the Royal Society A, 375, Article ID: 20150442. https://doi.org/10.1098/rsta.2015.0442

[6] Shankland, R.S. (1964) American Journal of Physics, 32, 16-35. https://doi.org/10.1119/1.1970063

[7] Sakharov, A.D. (1967) Doklady Akademii Nauk SSSR, 177, 70-71.

[8] Puthoff, H.E. (1989) Physical Review A, 39, 2333-2342. https://doi.org/10.1103/PhysRevA.39.2333

[9] Boccaletti, D., De Sabbata, V., Fortini, P. and Gualdi, C. (1970) Il Nuovo Cimento B (1965-1970), 70, 129-146. https://doi.org/10.1007/BF02710177

[10] DeWitt, B.C. (1967) Physical Review, 160, 1113-1147. https://doi.org/10.1103/PhysRev.160.1113

[11] DeWitt, B.C. (1967) Physical Review, 162, 1195-1239. https://doi.org/10.1103/PhysRev.162.1195

[12] DeWitt, B.C. (1967) Physical Review, 162, 1239-1256. https://doi.org/10.1103/PhysRev.162.1239

[13] dos Santos, W.C. (2016) Introduction to Einstein-Maxwell Equations and Rainich Conditions.

[14] Rovelli, C. (2011) Zakopane Lectures on Loop Gravity. Centre de Physique Theorique Luminy, Case 907, F-13288, EU.

[15] Engle, J., Pereira, R. and Rovelli, C. (2007) Physical Review Letters, 99, Article ID: 161301. https://doi.org/10.1103/PhysRevLett.99.161301

[16] Schwarzschild, K. (1916) Über das Gravitationsfeld eines Massenpunktes nach der Einsteinschen Theorie. Sitzungsberichte der Deutschen Akademie der Wissenschaften zu Berlin, Klasse fur Mathematik, Physik, und Technik, 189.

[17] Eddington, A.S. (1922) Mathematical Theory of Relativity. UP, Cambridge, 85-93.

[18] McDonald, K.T. (2013) Relativistic Harmonic Oscillator. Joseph Henry Laboratory, Princeton University, Princeton.

[19] Loveridge, C.L. (2004) Physical and Geometric Interpretation of the Ricci Tensor and Scalar Curvature.

[20] Wysin, G.M. (2011) Quantization of the Free Electromagnetic Field, Photons and Operators. Department of Physics, Kansas State University, Manhattan. 


\section{Appendix A}

The electromagnetic field tensor described here is only valid for gravity-free space. Any additions to the electromagnetic field tenser due to the effect of the Earth-like mass are small parameters. When these small parts of the electromagnetic Tensor are multiplied by the small terms describing the space-time curvature one obtains very small second-order terms that can be neglected. The $\mathrm{T}_{\mu \nu}$ component of the Electromagnetic Stress Tensor is given by:

$$
\begin{array}{ll}
\text { a) } \mathrm{T}_{\mu v}=\frac{1}{\mu_{\mathrm{o}}}\left[\mathrm{F}_{\mu \alpha} \eta^{\alpha \beta} \mathrm{F}_{\beta v}-\frac{1}{4} \eta_{\mu v} \mathrm{~F}^{\alpha \beta} \mathrm{F}_{\alpha \beta}\right] \quad \text { b) } \eta^{\mu \beta} \mathrm{F}_{\beta \alpha} \eta^{\alpha v}=\mathrm{F}^{\mu v}
\end{array}
$$

The contravariant Electromagnetic field tensor with components $F^{\mu v}$ and the covariant Electromagnetic field tensor with components $F_{\mu v}$ are:

$$
\text { a) } F^{\mu \rho}=\left[\begin{array}{cccc}
0 & -\frac{E_{1}}{c} & -\frac{E_{2}}{c} & -\frac{E_{3}}{c} \\
\frac{E_{1}}{c} & 0 & -B_{3} & B_{2} \\
\frac{E_{2}}{c} & B_{3} & 0 & -B_{1} \\
\frac{E_{3}}{c} & -B_{2} & B_{1} & 0
\end{array}\right] \quad \text { b) } F_{\rho v}=\left[\begin{array}{cccc}
0 & \frac{E_{1}}{c} & \frac{E_{2}}{c} & \frac{E_{3}}{c} \\
-\frac{E_{1}}{c} & 0 & -B_{3} & B_{2} \\
-\frac{E_{2}}{c} & B_{3} & 0 & -B_{1} \\
-\frac{E_{3}}{c} & -B_{2} & B_{1} & 0
\end{array}\right]
$$

and where $\eta^{\alpha \beta}$ is a component of the Minkowski Metric tensor for a Riemann space signature +--- .

$$
\eta^{\alpha \beta}=\left[\begin{array}{cccc}
1 & 0 & 0 & 0 \\
0 & -1 & 0 & 0 \\
0 & 0 & -1 & 0 \\
0 & 0 & 0 & -1
\end{array}\right]
$$

Substituting Equation (A2) and Equation (A3) into Equation (A1a)

$$
\begin{aligned}
& T_{\mu v}=\frac{1}{\mu_{o}}\left[\begin{array}{cccc}
\frac{E_{1}^{2}+E_{2}^{2}+E_{3}^{2}}{c^{2}} & -\frac{E_{2} B_{3}-E_{3} B_{2}}{c} & \frac{E_{1} B_{3}-E_{3} B_{1}}{c} & -\frac{E_{1} B_{2}-E_{2} B_{1}}{c} \\
-\frac{E_{2} B_{3}-E_{3} B_{2}}{2} & -\frac{E_{1}^{2}}{c^{2}}+B_{3}^{2}+B_{2}^{2} & -\frac{E_{1} E_{2}}{c^{2}}-B_{1} B_{2} & -\frac{E_{1} E_{3}}{c^{2}}-B_{1} B_{3} \\
\frac{E_{1} B_{3}-E_{3} B_{1}}{c} & -\frac{E_{1} E_{2}}{c^{2}}-B_{1} B_{2} & -\frac{E_{2}^{2}}{c^{2}}+B_{3}^{2}+B_{1}^{2} & -\frac{E_{2} E_{3}}{c^{2}}-B_{2} B_{3} \\
-\frac{E_{1} B_{2}-E_{2} B_{1}}{c} & -\frac{E_{1} E_{3}}{c^{2}}-B_{1} B_{3} & -\frac{E_{2} E_{3}}{c^{2}}-B_{2} B_{3} & -\frac{E_{3}^{2}}{c^{2}}+B_{2}^{2}+B_{1}^{2}
\end{array}\right] \\
& -\frac{1}{2}\left[\frac{\mathrm{E}_{1}^{2}+\mathrm{E}_{2}^{2}+\mathrm{E}_{3}^{2}}{\mathrm{c}^{2}}-\mathrm{B}_{1}^{2}-\mathrm{B}_{2}^{2}-\mathrm{B}_{3}^{2}\right]\left[\begin{array}{cccc}
1 & 0 & 0 & 0 \\
0 & -1 & 0 & 0 \\
0 & 0 & -1 & 0 \\
0 & 0 & 0 & -1
\end{array}\right]
\end{aligned}
$$

The Electric field and the Magnetic Flux density pseudo vectors of a Plane-wave propagating in the 3 direction are:

$$
\text { a) } \mathbf{E}=\hat{\mathbf{a}}_{1} E_{1} \exp \left(j \omega t-j \frac{\omega \mathrm{x}_{3}}{c}\right)
$$




$$
\text { b) } B_{2}=\frac{E_{1}}{c}
$$

where the Electric field vector is in the 1 direction. Substituting Equation (A5) into Equation (A4)

$$
\text { a) } T_{\mu \nu}=\varepsilon_{0} E_{1}^{2}\left[\begin{array}{cccc}
1 & 0 & 0 & -1 \\
0 & 0 & 0 & 0 \\
0 & 0 & 0 & 0 \\
-1 & 0 & 0 & 1
\end{array}\right] \quad \text { b) } T_{\mu \nu}=\varepsilon_{0} E_{1}^{2} e_{\mu \nu}
$$

where $\varepsilon_{0}$ is the dielectric constant of free isotropic space. 